\title{
FATORES DE RISCO NA GAGUEIRA DESENVOLVIMENTAL FAMILIAL E ISOLADA
}

\section{Risk factors in the familial and sporadic developmental stuttering}

\author{
Cristiane Moço Canhetti de Oliveira (1), Heloisa Aparecida de Souza (2), \\ Ana Claudia dos Santos ${ }^{(3)}$, Denise Cunha ${ }^{(4)}$, Célia Maria Giacheti ${ }^{(5)}$
}

\begin{abstract}
RESUMO
Objetivo: investigar e comparar os achados dos fatores de risco para a cronicidade da gagueira em crianças com gagueira desenvolvimental familial e isolada. Método: participaram 60 crianças de ambos os gêneros, divididas em dois grupos: $\mathrm{Gl}-30$ crianças com gagueira desenvolvimental familial; Gll - 30 crianças com gagueira desenvolvimental isolada. A coleta de dados foi realizada por meio do Protocolo de Risco para a Gagueira do Desenvolvimento - PRGD (Andrade, 2006), que considera os seguintes fatores de risco: idade, gênero, tipo de surgimento e tempo de duração das disfluências, tipologia das disfluências, fatores comunicativos e qualitativos associados, histórico mórbido pré, peri e pós natal, fatores estressantes que ocorreram próximo ao surgimento do distúrbio, histórico familial, reação pessoal, familiar e social e atitudes familiares. Resultados: quando o grupo I (GI) foi comparado com o grupo II (GII), a única diferença estatisticamente significante foi com relação aos fatores estressantes que ocorreram próximo ao surgimento do distúrbio. Conclusão: os resultados confirmam a natureza complexa da gagueira, bem como a necessidade de se investigar os vários fatores considerados como de risco para o distúrbio, com intuito de melhorar a compreensão de suas possíveis etiologias.
\end{abstract}

DESCRITORES: Gagueira; Fatores de Risco; Genética; Fonoaudiologia

\section{INTRODUÇÃO}

A gagueira desenvolvimental é um distúrbio da comunicação que se inicia na infância e é

(1) Fonoaudióloga; Docente do Departamento de Fonoaudiologia da Faculdade de Filosofia e Ciências da Universidade Estadual Paulista, UNESP, Marília, SP, Brasil; Doutora em Ciências Biológicas na área de Genética pelo Instituto de Biociências da Universidade Estadual Paulista, UNESP, Botucatu, SP.

(2) Fonoaudióloga graduada pela Faculdade de Filosofia e Ciências da Universidade Estadual Paulista, UNESP, Marília, SP, Brasil.

(3) Graduanda em Fonoaudiologia pela Faculdade de Filosofia e Ciências da Universidade Estadual Paulista, UNESP, Marília, SP, Brasil.

(4) Graduanda em Fonoaudiologia pela Faculdade de Filosofia e Ciências da Universidade Estadual Paulista, UNESP, Marília, SP, Brasil.

(5) Fonoaudióloga; Professor Adjunto do Departamento de Fonoaudiologia da Faculdade de Filosofia e Ciências da Universidade Estadual Paulista, UNESP, Marília, SP, Brasil; Livre Docente pela UNESP, Marília, SP.

Conflito de interesses: inexistente caracterizada por rupturas involuntárias na fluência da fala. A produção da fala disfluente, tensa e com esforço, pode acarretar conseqüências sociais e emocionais no indivíduo que gagueja, prejudicando sua qualidade de vida. Neste sentido, investigações que propiciem maiores esclarecimentos sobre os fatores que interferem no surgimento da gagueira, se fazem necessárias para compreender sua etiologia e prevenir o seu desenvolvimento, suas conseqüências pessoais, e, ainda, favorecer o resultado terapêutico.

Embora a etiologia da gagueira ainda não tenha sido precisamente identificada, diversas pesquisas ${ }^{1-3}$ têm mostrado que fatores genéticos estão envolvidos na transmissão da suscetibilidade do distúrbio. As principais evidências que sustentam a predisposição genética na gagueira são: sua maior prevalência entre os parentes de gagos quando comparados com grupo controle ${ }^{1}$, a maior concordância do distúrbio entre os gêmeos monozigóticos do que gêmeos dizigóticos ${ }^{4}$, e os estudos de adoção que sugerem que a gagueira da criança está mais 
relacionada com a gagueira dos pais biológicos que com a dos pais adotivos ${ }^{2}$.

A gagueira desenvolvimental familial foi descrita na literatura como um possível subgrupo do distúrbio, cuja etiologia parece ser primariamente de origem genética ${ }^{1,4-6}$, e que geralmente inicia-se de forma gradual, sem a presença de fatores estressantes ${ }^{7}$. Aproximadamente metade dos casos de gagueira desenvolvimental persistente apresenta histórico familial positivo do distúrbio ${ }^{6}$. O outro subgrupo de gagueira com origem na infância e sem predisposição genética é descrito como gagueira desenvolvimental isolada.

A gagueira é um distúrbio multifatorial resultante de inúmeros fatores de risco ${ }^{8}$. O início predominante do distúrbio em idade pré-escolar sugere que fatores múltiplos relevantes são causados no processo desenvolvimental ${ }^{9}$. O alinhamento desses fatores por um período (ou períodos), durante seus diferentes ritmos de desenvolvimento, pode contribuir para o surgimento da gagueira ${ }^{9}$.

Assim sendo, na história clínica da gagueira é de extrema relevância que se conheça os fatores considerados como de risco para a cronicidade do distúrbio. Dentre estes fatores estão a idade, o gênero, o tipo de surgimento e o tempo de duração das disfluências, a tipologia das disfluências, os fatores comunicativos e qualitativos associados, o histórico mórbido pré, peri e pós natal, o histórico familial, os fatores estressantes que ocorreram próximo ao surgimento do distúrbio, a reação pessoal, familiar e social, e atitudes familiares ${ }^{10}$.

As disfluências freqüentemente aparecem no período de aquisição da linguagem, especialmente entre 2 e 5 anos ${ }^{11-14}$. A idade média do surgimento dessas interrupções na fala é de 30 meses $^{7,15}$, e com 4 anos de idade a maioria das crianças gagas já manifestou este distúrbio ${ }^{4}$. As disfluências gagas iniciam-se nos anos pré-escolares, prejudicando a comunicação, e podem ser acompanhadas de sentimentos e emoções negativas, como timidez, medo relacionado à fala, além de ansiedade em determinadas situações ${ }^{16}$.

A recuperação espontânea da gagueira ocorre principalmente durante os primeiros anos de vida ${ }^{17-19} \mathrm{e}$, geralmente, no intervalo dos primeiros 12 meses após o surgimento do distúrbio. Portanto, quanto maior o tempo de duração das disfluências, menor a possibilidade de ocorrer a recuperação espontânea da gagueira.

O conhecimento da tipologia das disfluências é importante para caracterizar as rupturas na fala, identificando-as como comuns ou gagas, o que contribuirá para a realização do diagnóstico. A sintomatologia da gagueira é caracterizada por excessiva repetição de sons e palavras monossilábicas, prolongamentos e bloqueios ${ }^{12}$. Pesquisa recente mostrou que os pais de crianças com gagueira podem ser precisos e confiáveis na identificação dessas disfluências na fala de seus filhos ${ }^{20}$. Crianças com gagueira apresentam uma alta taxa de descontinuidade de fala e de disfluências gagas, geralmente maiores que $10 \%$ e $3 \%$, respectivamente ${ }^{21}$.

Fatores qualitativos associados, como tensão corporal ou facial, aumento da taxa de elocução, incoordenação respiratória e/ou variação da intensidade e freqüência vocal, podem acompanhar as disfluências de crianças com gagueira ${ }^{17}$. Nos quadros típicos de risco e alto risco para a gagueira, é comum a presença de fatores comunicativos associados, como alterações fonológicas, miofuncionais, de compreensão, memória, entre outras ${ }^{10}$. Por outro lado, alguns estudos ${ }^{9,15}$ acreditam que o início da gagueira em crianças pequenas pode estar relacionado a escores aumentados de vocabulário.

Apesar dos fatores psicossociais estressantes serem citados na literatura como um dos fatores de risco para o desenvolvimento da gagueira, um estudo não mostrou diferença em termos estatísticos nos grupos de baixo risco, risco e alto risco ${ }^{10}$.

Alguns comportamentos lingüísticos e paralingüísticos inadequados dos familiares em relação à gagueira infantil, como o não respeito à troca de turnos, o oferecimento de "pistas e truques" para evitar as disfluências, as atitudes paternas não verbais e correções, críticas ou modificação das frases das crianças, são comumente encontrados ${ }^{22}$. Uma investigação comparou as atitudes familiares em crianças de baixo risco, risco e alto risco para o desenvolvimento da gagueira e constatou que quanto maior o risco, pior era a qualidade destes comportamentos, havendo diferença estatisticamente significante entre os grupos ${ }^{10}$. Outro estudo encontrou que a taxa de elocução aumentada das mães estavam estatisticamente associadas com a maior gravidade da gagueira de seus filhos ${ }^{23}$. Crianças com gagueira são mais prováveis de serem influenciadas pelas características temporais da fala de seus pais quando comparadas com crianças fluentes, por exemplo, quando os pais interrompiam a criança, esta apresentava uma tendência de interromper no turno subseqüente numa duração de tempo semelhante ${ }^{24}$. A importância da atitude familiar também ficou evidente pelo estudo, que mostrou que a terapia realizada pela interação pais-criança pode reduzir a gagueira da criança pré-escolar ${ }^{25}$.

Quanto à reação social, encontramos que as reações negativas dos interlocutores da criança com gagueira podem prejudicar o prognóstico terapêutico e sua habilidade de comunicação ${ }^{26}$. 
A gagueira pode eliciar respostas negativas dos pares de comunicação e afetar outras interações sociais da criança ${ }^{27}$.

O Protocolo de Risco para a Gagueira do Desenvolvimento ${ }^{10}$ considera estes fatores de risco e apresenta uma pontuação especifica de acordo com a importância de cada um. Este procedimento é de extrema relevância na área da fluência e auxilia o diagnóstico da disfluência/gagueira infantil. Porém, investigações que compararam estes fatores em grupos de crianças com gagueira desenvolvimental familial e isolada são escassas.

Portanto, o objetivo deste trabalho foi investigar e comparar os achados dos fatores de risco para a cronicidade da gagueira em crianças com gagueira desenvolvimental familial e com gagueira desenvolvimental isolada.

\section{MÉTODO}

Participaram dessa pesquisa 60 crianças matriculadas em instituição que atende crianças com gagueira na faixa etária de 3.0 a 11.11 anos de idade, de ambos os gêneros ( 45 do gênero masculino e 15 do gênero feminino), cujos pais concordaram, por meio de assinatura do Termo de Consentimento, com a realização dos procedimentos propostos para realização do estudo.

Os participantes dessa investigação foram divididos em dois grupos: o grupo I (GI) foi composto por 30 crianças com gagueira desenvolvimental familial, idade média de 6,3 anos, sendo 23 do gênero masculino e 7 do gênero feminino; e o grupo II (GII) com 30 crianças com gagueira desenvolvimental isolada, idade média de 5,4 anos, sendo 22 do gênero masculino e 8 do gênero feminino.

A Tabela 1 mostra que não houve diferenças significantes na faixa etária e no gênero entre os grupos estudados. A razão sexual no grupo de gagueira desenvolvimental familial foi de 3,3 meninos para 1 menina, enquanto que no grupo de gagueira desenvolvimental isolada foi de 2,7 meninos para 1 menin

Tabela 1 - Distribuição dos participantes nos grupos quanto à faixa etária e gênero

\begin{tabular}{cccccccc}
\hline \multirow{2}{*}{ GRUPO } & \multicolumn{9}{c}{ Faixa etária } & \multicolumn{2}{c}{ Gênero } \\
\cline { 2 - 7 } & $\begin{array}{c}\mathbf{3 . 0} \text { a 4.11 } \\
\text { anos }\end{array}$ & $\begin{array}{c}\mathbf{5 . 0} \text { a } \mathbf{6 . 1 1} \\
\text { anos }\end{array}$ & $\begin{array}{c}\mathbf{7 . 0} \text { a 9.11 } \\
\text { anos }\end{array}$ & $\begin{array}{c}\mathbf{1 0 . 0} \text { a 11.11 } \\
\text { anos }\end{array}$ & masculino & feminino \\
\hline \multirow{2}{*}{ GI } & 3 & 7 & 12 & 8 & 23 & 7 \\
& $10 \%$ & $23,3 \%$ & $40 \%$ & $26,7 \%$ & $76,7 \%$ & $23,3 \%$ \\
\hline \multirow{2}{*}{ Gll } & 4 & 11 & 13 & 2 & 22 & 8 \\
& $13,3 \%$ & $36,7 \%$ & $43,3 \%$ & $6,7 \%$ & $73,3 \%$ & $26,7 \%$ \\
\hline \multirow{2}{*}{ Total } & 7 & 18 & 25 & 10 & 45 & 15 \\
& $11,7 \%$ & $30 \%$ & $41,6 \%$ & $16,7 \%$ & $75 \%$ & $25 \%$ \\
\hline $\begin{array}{c}\text { p-valor } \\
(\mathbf{p}<\mathbf{0 , 0 5})\end{array}$ & \multicolumn{2}{c}{$\mathrm{p}=0,197$} & & $\mathrm{p}=0,766$ \\
\hline
\end{tabular}

Análise estatística: Teste de Qui-quadrado

Os requisitos de inclusão dos participantes foram: idade entre 03 e 11 anos e 11 meses, ser falante nativo do português brasileiro, não apresentar déficit neurológico, auditivo, cognitivo e/ou visual, apresentar queixa de gagueira por parte dos pais ou familiares.

Os critérios de exclusão dos participantes foram: apresentar qualquer distúrbio neurológico genético ou não, tais como distonia, doenças extras piramidais, deficiência mental, epilepsia, transtorno do déficit de atenção e hiperatividade (TDAH); sintomas ou condições psiquiátricas; apresentar alterações de comunicação oral não compatíveis com a idade, com exceção à gagueira; apresentar perda auditiva condutiva ou neurossensorial, e; outras condições pertinentes que poderiam gerar erros no diagnóstico.

O diagnóstico de gagueira foi estabelecido segundo os critérios:

- presença de no mínimo 3\% de disfluências gagas;

- receber 11 pontos ou mais (critério mínimo para a gravidade da gagueira ser classificada como "leve") no Stuttering Severity Instrument - 3 $(\mathrm{SSI}-3)^{28}$.

Para o GI, as crianças apresentaram pelo menos algum outro familiar com gagueira, e no 
Gll, nenhum dos participantes apresentou queixa pessoal e/ou familiar de gagueira.

Inicialmente, os familiares dos participantes receberam informações sobre os objetivos do estudo e explicação dos procedimentos que seriam realizados, além do que, deram consentimento, por escrito, para a participação na pesquisa.

A coleta de dados foi realizada por meio da aplicação do Protocolo de Risco para a Gagueira do Desenvolvimento - PRGD ${ }^{10}$. O Protocolo foi realizado com os pais ou familiares das crianças, com a finalidade de detectar os fatores de risco para a cronicidade da gagueira, como: idade, gênero, tempo de cronicidade das disfluências, tipo de início e tipologia das disfluências, fatores estressantes físicos e/ou emocionais que ocorreram próximo ao surgimento das disfluências, fatores genéticos, fatores comunicativos e qualitativos associados, presença de reações negativas da criança, da família e do meio social e atitude familiar ${ }^{10}$.

Os dados dos antecedentes familiares para realizar o heredograma foram coletados durante a história clínica. Os familiares adultos de primeiro grau foram questionados sobre o padrão de fluência de seus parentes, e sobre a existência de alguém na família que eles soubessem apresentar gagueira. Para possibilitá-los a responder estas questões, a entrevistadora apresentou uma definição padronizada de gagueira ${ }^{29}$ e ofereceu exemplos que pudessem ilustrá-las. Com o intuito de obter informações precisas, os familiares foram encorajados a checar e a confirmar com outros parentes, informações e histórias de gagueira.

Para a avaliação específica da gagueira foi realizada gravação de fala espontânea, utilizando-se uma filmadora Digital Sony. As gravações foram transcritas e, a seguir, as disfluências foram mapeadas em comuns e gagas, segundo protocolo específico ${ }^{30}$.

Os participantes foram separados em dois grupos: Gl com gagueira desenvolvimental familial e, Gll com gagueira desenvolvimental isolada, de acordo com os dados da história familial.

Após a realização do protocolo, a família recebeu a devolutiva dos resultados obtidos. Informações sobre gagueira foram oferecidas por meio de orientações e instruções, com o auxílio de um informe, e, os casos foram encaminhados para a terapia fonoaudiológica no próprio Centro onde foi desenvolvido o projeto.

Este estudo foi aprovado pelo Comitê de Ética em Pesquisa da Faculdade de Filosofia e Ciências da UNESP - Parecer CEP № 3490/2008 CEP - 023/2006.

Para a obtenção dos resultados, foi utilizado o programa SPSS (Statistical Package for Social Sciences), em sua versão 13.0. O Teste de Quiquadrado foi aplicado, com o intuito de compararmos os resultados e verificarmos possíveis diferenças entre os grupos estudados. Adotamos o nível de significância de 5\%.

\section{RESULTADOS}

Os resultados apresentados na Tabela 2 representam as informações sobre as disfluências manifestadas pelas crianças, segundo a opinião dos seus pais ou familiares. Como pode ser observado, não houve diferença estatística significante quanto ao tipo de surgimento das disfluências, tempo de surgimento e tipologia das disfluências.

Tabela 2 - Distribuição dos participantes nos grupos quanto às disfluências, referente ao tipo e tempo de surgimento e tipologia das disfluências

\begin{tabular}{cccccccccc}
\hline \multirow{2}{*}{ GRUPO } & \multicolumn{2}{c}{$\begin{array}{c}\text { Tipo de surgimento das } \\
\text { disfluências }\end{array}$} & \multicolumn{2}{c}{$\begin{array}{c}\text { Tempo de } \\
\text { surgimento das } \\
\text { disfluências }\end{array}$} & \multicolumn{2}{c}{ Tipologia das disfluências } \\
\cline { 2 - 9 } & súbito & cíclico & $\begin{array}{c}\text { persis- } \\
\text { tente }\end{array}$ & $\begin{array}{c}\mathbf{6 - 1 2} \\
\text { meses }\end{array}$ & $\begin{array}{c}\mathbf{+ 1 2} \\
\text { meses }\end{array}$ & comum & mista & gaga \\
\hline \multirow{2}{*}{ GI } & 11 & 6 & 13 & 0 & 30 & 1 & 5 & 24 \\
& $36,7 \%$ & $20 \%$ & $43,3 \%$ & $0 \%$ & $100 \%$ & $3,3 \%$ & $16,7 \%$ & $80 \%$ \\
\hline \multirow{2}{*}{ GII } & 5 & 10 & 15 & 3 & 27 & 5 & 2 & 23 \\
& $16,7 \%$ & $33,3 \%$ & $50 \%$ & $10 \%$ & $90 \%$ & $16,7 \%$ & $6,7 \%$ & $76,6 \%$ \\
\hline \multirow{2}{*}{ Total } & 16 & 16 & 28 & 3 & 57 & 6 & 7 & 47 \\
& $26,7 \%$ & $26,7 \%$ & $46,6 \%$ & $5 \%$ & $95 \%$ & $10 \%$ & $11,7 \%$ & $78,3 \%$ \\
\hline $\begin{array}{c}\text { p-valor } \\
\text { (p<0,05) }\end{array}$ & & $p=0,183$ & & $p=0,076$ & & $p=0,137$ & \\
\hline
\end{tabular}

Análise estatística: Teste de Qui-quadrado 
A Tabela 3 apresenta os resultados referentes aos fatores comunicativos e qualitativos associados à gagueira dos participantes distribuídos nos dois grupos. Observa-se que o grupo com gagueira desenvolvimental isolada (GII) apresentou uma pequena tendência a manifestar maior número de fatores comunicativos e qualitativos associados à gagueira do que o grupo com gagueira desenvolvimental familial (GI).

Tabela 3 - Distribuição dos participantes nos grupos quanto aos fatores comunicativos e qualitativos associados à gagueira

\begin{tabular}{ccccccc}
\hline \multirow{2}{*}{ GRUPO } & \multicolumn{2}{c}{ Fatores comunicativos associados } & \multicolumn{2}{c}{ Fatores qualitativos associados } \\
\cline { 2 - 7 } & Não tinha & Tinha 1 & $\begin{array}{c}\text { Tinha } \\
\text { mais de 1 }\end{array}$ & Não tinha & Tinha 1 & $\begin{array}{c}\text { Tinha } \\
\text { mais de 1 }\end{array}$ \\
\hline \multirow{2}{*}{ GI } & 10 & 11 & 9 & 2 & 10 & 18 \\
& $33,3 \%$ & $36,7 \%$ & $30 \%$ & $6,7 \%$ & $33,3 \%$ & $60 \%$ \\
\hline \multirow{2}{*}{ GII } & 10 & 8 & 12 & 4 & 4 & 22 \\
& $33,3 \%$ & $26,7 \%$ & $40 \%$ & $13,3 \%$ & $13,3 \%$ & $73,4 \%$ \\
\hline Total & 20 & 19 & 21 & 6 & 14 & 40 \\
\hline $\begin{array}{c}\text { p-valor } \\
\text { (p<0,05) }\end{array}$ & $33,3 \%$ & $31,7 \%$ & $35 \%$ & $10 \%$ & $23,3 \%$ & $66,7 \%$ \\
\hline Análise estatística: Teste de Qui-quadrado & $\mathrm{p}=0,637$ & & & $\mathrm{p}=0,132$ & \\
\hline
\end{tabular}

Análise estatística: Teste de Qui-quadrado

Procurando verificar a presença ou não de uma diferença estatística entre Gl e Gll quanto ao histórico mórdibo pré-peri-pós-natal, e presença de componentes estressantes próximos ao surgimento das disfluências, a aplicação do Teste de Qui-quadrado foi realizada (Tabela 4). Os resultados indicaram que os grupos não se diferenciam estatisticamente quanto ao histórico mórdibo pré-peri-pós-natal. Porém, a ocorrência de fatores estressantes próximos ao surgimento das disfluências foi maior no grupo com gagueira desenvolvimental isolada (GII), sendo essa diferença estatisticamente relevante em relação ao grupo com gagueira desenvolvimental familial (GI).

Tabela 4 - Distribuição dos participantes nos grupos quanto ao histórico mórdibo pré-peri-pós-natal e presença de componentes estressantes próximos ao surgimento das disfluências

\begin{tabular}{|c|c|c|c|c|c|c|c|}
\hline \multirow[b]{2}{*}{ GRUPO } & \multicolumn{3}{|c|}{ Histórico mórdibo pré-peri-pós-natal } & \multicolumn{4}{|c|}{$\begin{array}{c}\text { Componentes estressantes próximos ao } \\
\text { surgimento das disfluências }\end{array}$} \\
\hline & Não tinha & $\begin{array}{c}\text { Somente } 1 \\
\text { fator sem } \\
\text { sequela }\end{array}$ & $\begin{array}{l}\text { + de } 1 \text { fator } \\
\text { ou com } \\
\text { suspeita de } \\
\text { seqüela }\end{array}$ & Não tinha & $\begin{array}{l}\text { Até } 25 \\
\text { pontos }\end{array}$ & $\begin{array}{c}25-50 \\
\text { pontos }\end{array}$ & $\begin{array}{c}\text { Mais de } 50 \\
\text { pontos }\end{array}$ \\
\hline \multirow{2}{*}{ GI } & 21 & 4 & 5 & 6 & 11 & 10 & 3 \\
\hline & $70 \%$ & $13,3 \%$ & $16,7 \%$ & $20 \%$ & $36,7 \%$ & $33,3 \%$ & $10 \%$ \\
\hline \multirow{2}{*}{ GII } & 23 & 5 & 2 & 4 & 5 & 8 & 13 \\
\hline & $76,6 \%$ & $16,7 \%$ & $6,7 \%$ & $13,3 \%$ & $16,7 \%$ & $26,7 \%$ & $43,3 \%$ \\
\hline \multirow{2}{*}{ Total } & 44 & 9 & 7 & 10 & 16 & 18 & 16 \\
\hline & $73,3 \%$ & $15 \%$ & $11,7 \%$ & $16,6 \%$ & $26,7 \%$ & $30 \%$ & $26,7 \%$ \\
\hline $\begin{array}{l}p \text {-valor } \\
(p<0,05)\end{array}$ & \multicolumn{3}{|c|}{$p=0,613$} & \multicolumn{4}{|c|}{$p=0,028^{*}$} \\
\hline
\end{tabular}

Análise estatística: Teste de Qui-quadrado 
Os resultados apresentados na Tabela 5 representam a comparação entre os grupos quanto à reação e atitude familiar, reação pessoal e social. Como pode ser observado, não houve diferença estatística significante nesses aspectos. Nota-se que a maioria de Gl e Gll apresentou reações familiar e social adequadas. No entanto, a maior parte da população participante revelou atitude familiar e reação pessoal inadequadas, nos dois grupos estudados.

Tabela 5 - Distribuição dos participantes nos grupos quanto à reação e atitude familiar, reação pessoal e reação social

\begin{tabular}{|c|c|c|c|c|c|c|c|c|c|c|c|}
\hline \multirow{2}{*}{ GRUPO } & \multicolumn{3}{|c|}{ Reação familiar } & \multicolumn{2}{|c|}{ Atitude familiar } & \multicolumn{3}{|c|}{ Reação pessoal } & \multicolumn{3}{|c|}{ Reação social } \\
\hline & + & alerta & - & alerta & - & + & Alerta & - & + & alerta & - \\
\hline \multirow{2}{*}{ GI } & 16 & 6 & 8 & 1 & 29 & 1 & 2 & 27 & 16 & 10 & 4 \\
\hline & $53,3 \%$ & $20 \%$ & $26,7 \%$ & $3,3 \%$ & $96,7 \%$ & $3,3 \%$ & $6,7 \%$ & $90 \%$ & $53,3 \%$ & $33,3 \%$ & $13,4 \%$ \\
\hline \multirow{2}{*}{ Gll } & 15 & 7 & 8 & 2 & 28 & 1 & 1 & 28 & 14 & 7 & 9 \\
\hline & $50 \%$ & $23,3 " \%$ & $26,7 \%$ & $6,7 \%$ & $93,3 \%$ & $3,3 \%$ & $3,3 \%$ & $93,4 \%$ & $46,7 \%$ & $23,3 \%$ & $30 \%$ \\
\hline \multirow{2}{*}{ Total } & 31 & 13 & 16 & 3 & 57 & 2 & 3 & 55 & 30 & 17 & 13 \\
\hline & $51,6 \%$ & $21,7 \%$ & $26,7 \%$ & $5 \%$ & $95 \%$ & $3,3 \%$ & $5 \%$ & $91,7 \%$ & $50 \%$ & $28,3 \%$ & $21,7 \%$ \\
\hline $\begin{array}{c}\text { p-valor } \\
(p<0,05)\end{array}$ & \multicolumn{3}{|c|}{$p=0,947$} & \multicolumn{2}{|c|}{$p=0,554$} & \multicolumn{3}{|c|}{$p=0,839$} & \multicolumn{3}{|c|}{$p=0,274$} \\
\hline
\end{tabular}

Análise estatística: Teste de Qui-quadrado

\section{DISCUSSÃO}

O presente estudo foi conduzido para investigar os fatores de risco para a gagueira desenvolvimental, e comparar os grupos de crianças com gagueira familial com o grupo com gagueira isolada. Achados interessantes resultaram desta investigação.

$\mathrm{Na}$ análise estatística, constatamos que não houve diferença significante, quando os dois grupos foram comparados com relação à idade, gênero, tempo de cronicidade das disfluências, tipo de início e tipologia das disfluências, fatores estressantes físicos, fatores comunicativos e qualitativos associados, presença de reações negativas da criança, da família e do meio social e atitude familiar. Notase, portanto, que o subtipo de gagueira desenvolvimental familial parece não distinguir com relação a estes aspectos, quando comparados com o grupo de gagueira desenvolvimental isolada. No entanto, os dados desta investigação indicaram que esses fatores, juntos, podem contribuir para o surgimento e persistência da gagueira desenvolvimental, enfatizando a natureza multidimensional do distúrbio, como já descrito na literatura ${ }^{18}$.

Na comparação estatística entre Gl e Gll, a única diferença significante foi com relação aos fatores estressantes que ocorreram próximo ao surgimento do distúrbio. No grupo de gagueira desenvolvimental isolada houve uma tendência de ocorrer mais fatores estressantes e de maior pontuação do que no grupo com gagueira desenvolvimental familial, corroborando estudo prévio ${ }^{7}$.

Os resultados desse estudo concordam que fatores genéticos parecem atuar em conjunto com outros fatores, que numa interação dinâmica e complexa, ainda não esclarecida pode justificar o surgimento do distúrbio, conforme descrito na literatura ${ }^{8}$. Para desencadear as manifestações da gagueira, pode ser que, crianças sem predisposição genética para a desordem necessitem de mais fatores de risco, ou de fatores de risco mais relevantes em seu desenvolvimento. Enquanto que o grupo de crianças que apresenta histórico de gagueira na família manifesta maior risco de recorrência do distúrbio, necessitando de menos fatores de risco associados para desencadear o surgimento da gagueira. Conforme apontado na literatura, a presença de histórico de gagueira na família representa um alto risco para o distúrbio ${ }^{9,31}$.

Nossos dados não mostraram diferença estatisticamente significante, quando os grupos foram comparados quanto ao histórico mórbido pré-peripós-natal. Esses achados se diferenciaram do estudo que descreveu que danos cerebrais precoces, traumas ou doenças no nascimento ou no desenvolvimento podem justificar o surgimento da gagueira desenvolvimental isolada ${ }^{5}$.

Os resultados dessa investigação sugerem que, independente da presença de aspectos genéticos, a gagueira desenvolvimental apresenta maior 
prevalência no gênero masculino, como citado por vários autores ${ }^{4,12,18,29}$.

Apesar da gagueira freqüentemente surgir antes dos 4 anos de idade ${ }^{4}$, os pais ou responsáveis geralmente demoraram para procurar o atendimento fonoaudiológico nos dois grupos de participantes. Dados estes confirmados pelas idades das crianças que buscaram atendimento e o tempo de surgimento das disfluências. Este achado sugere que fonoaudiólogos ainda não recebem precocemente crianças para avaliação, diagnóstico e intervenção nos casos de gagueira, familial ou isolada, o que implica, clinicamente, em atendimentos tardios. $\mathrm{Na}$ tentativa de resolver este problema, profissionais poderiam desenvolver ações no sentido de esclarecer a população em geral, pais, educadores e também profissionais da saúde para que os encaminhamentos fossem mais precoces, pelo menos que profissionais especialistas da área pudessem realizar o diagnóstico adequado e definir a conduta.

O tipo de início da gagueira mais freqüente nos dois grupos participantes foi o persistente ou gradual. Portanto, nossos achados demonstraram que este dado pode ser característico do grupo de crianças com gagueira desenvolvimental, e não somente do grupo de gagueira desenvolvimental familial. Na literatura, encontramos esse tipo de início como característico da gagueira familial ${ }^{7}$.

A ocorrência de disfluências gagas é um critério para o diagnóstico de gagueira ${ }^{4,7,12,18,21}$, e, por esse motivo, os resultados foram semelhantes entre os grupos. Com relação à presença de disfluências por mais de 12 meses, também é típico da gagueira desenvolvimental persistente, independente de sua natureza ${ }^{17,18}$.

Os resultados dessa pesquisa sugerem que a reação e atitude familiar independem da presença ou não de outro parente com gagueira. No entanto, não foi objetivo do estudo investigar os fatores de risco relacionados com o grau de parentesco do familiar afetado. Sabe-se que o risco de recorrência é maior quando o familiar afetado é de primeiro grau, portanto, investigações futuras poderão analisar a reação e atitude familiar no grupo de gagueira desenvolvimental familial com afetados de primeiro grau, em comparação ao grupo com afetados de segundo e terceiro graus.
Acreditamos que os fatores de risco também podem ser analisados com o auxílio de avaliações específicas, para averiguar determinados aspectos relacionados ao distúrbio atualmente destacados na literatura como, a consciência da gagueira ${ }^{32}$, o comportamento de pessoas que gaguejam ${ }^{33} \mathrm{a}$ ansiedade ${ }^{34,35}$, entre outros.

$\mathrm{Na}$ literatura encontramos muitas investigações que comparam características de crianças com gagueira e crianças fluentes, no entanto, existe uma tendência atual em delinear possíveis subtipos de gagueira ${ }^{36}$. Porém, até o momento, investigações descritivas ou mesmo comparativas desses subtipos são escassas. Neste sentido, esta pesquisa apresenta dados relevantes que poderão auxiliar em futuros estudos sobre os subtipos de gagueira.

\section{CONCLUSÃO}

Pode-se sugerir que os fatores de risco pesquisados nos dois grupos de crianças com gagueira são similares, independentes do histórico familial. Os fatores que mais se destacaram nos dois grupos foram: o gênero masculino; o surgimento persistente, a duração de mais de 12 meses das disfluências; a presença da tipologia gaga e de fatores qualitativos associados às disfluências, e; a atitude familiar e reação pessoal negativa.

Quando os dois grupos foram comparados, quanto aos fatores de risco, encontramos apenas que os fatores estressantes próximos ao surgimento das disfluências apresentaram diferença estatisticamente significante. Acredita-se que nos casos em que não ocorre histórico familial positivo para gagueira, são necessários mais fatores de risco, por isso o grupo de gagueira desenvolvimental isolada pode ter apresentado significantemente mais fatores estressantes, em relação ao grupo de gagueira familial.

\section{AGRADECIMENTOS}

A Pró-Reitoria de Extensão - PROEX da UNESP pela concessão de fomento à pesquisa realizada. 


\section{ABSTRACT}

Purpose: to investigate and compare the risk factors for stuttering between children with familial developmental stuttering and children with sporadic developmental stuttering. Method: 60 children of both genders with stuttering took part, divided in two groups: $\mathrm{Gl}-30$ children with familial developmental stuttering; GII - 30 children with sporadic developmental stuttering. Data were gathered through the Protocol of Risk for the Developmental Stuttering - PRGD (Andrade, 2006), which considers the following factors: age; gender; manner of onset and time of duration for the disfluencies; typology of the disfluencies; associated communicative and qualitative factors; physical and emotional stress; family history concerning stuttering; personal, familiar and social reaction, and familiar attitudes. Results: when Group I (GI) was compared to Group II (GII), the only statically difference was related to emotional stress that occurred near the onset of the disorder. Conclusion: the results confirm the complexity of stuttering, as well as the need for investigating the risk factors for this disorder in order to improve the understanding of its possible etiologies.

KEYWORDS: Stuttering; Risk Factors; Genetics; Speech, Language and Hearing Sciences

\section{REFERÊNCIAS BIBLIOGRÁFICAS}

1. Yairi E, Ambrose NG, Cox N. Genetics of stuttering: a critical review. J Speech Lang Hear Res. 1996;39:771-84.

2. Felsenfeld S. Progress and needs in the genetics of stuttering. J Fluency Disord. 1996;21:77-103.

3. Canhetti-Oliveira, MC, Richieri-Costa A. A study of familial stuttering. Amer J Med Genet. 2006;140A: 2139-41.

4. Bloodstein O. A handbook on stuttering. Chicago: National Easter Seal Society; 1995.

5. Poulos MG, Webster WG. Family history as a basis for subgrouping people who stutter. J Speech Hear Res. 1991;34:5-10.

6. Drayna D, Kilshaw J, Kelly J. The sex ratio in familial persistent stuttering. Am J Hum Genet.1999;65:1473-5.

7. Yairi E, Ambrose N. Onset of stuttering in preschool children: select factors. J Speech Lang Hear Res. 1992;35(4):783-8.

8. Smith A, Kelly E. Stuttering: A dynamic, multifactorial model. In: Curlee RF, Siegel GM, editors. Nature and treatment of stuttering: New directions. Needham Heights: Allyn \& Bacon; 1997. p. 204-17.

9. Yairi E, Ambrose NG. Early childhood stuttering: for clinicians by clinicians. Austin: Pro-Ed; 2005.

10. Andrade CRF. Gagueira infantil: risco, diagnóstico e programas terapêuticos. Barueri: Pró Fono; 2006.

11. Bloodstein O. Some empirical observations about early stuttering: A possibly link to language development. J Commun Disord. 2006;30:185-91.
12. Wittke-Thompson JK, Ambrose N, Yairi EC, Cook EH, Ober C. et al. Genetic studies of stuttering in a founder population. J Fluency Disord. 2007;32:33-50.

13. Wagovich SA, Hall NE, Cliffor BA. Speech disruptions in relation to language growth in children who stutter: An exploratory study. J Fluency Disord, 2009. doi: 10.1016/j.jfludis.2009.09.004.

14. Logan KJ, Mullins MS, Jones KM. The depiction of stuttering in contemporary juvenile fiction: implications for clinical practice. Psychol Sch. 2008;45(7):609-26.

15. Reilly $S$, Onslow $M$, Packman A, Wake $M$, Bavin EL, Prior M, Eadie P, Cini E, Bolzonello C, Ukoumunne OC. Predicting stuttering onset by age of 3 years: A prospective, community cohort study. Pediatrics. 2009;123:270-7.

16. Logan KJ, Mullins MS, Jones KM. The depiction of stuttering in contemporary juvenile fiction: implications for clinical practice. Psychol Sch. 2008;45(7):609-26.

17. Yairi E, Ambrose N, Niermann R. The early months of stuttering: a developmental study. J Speech Lang Hear Res. 1993;36(3):521-8.

18. Yairi E, Ambrose NG, Paden EP, Throneburg RN. Predictive factors of persistence and recovery: pathways of childhood stuttering. J Commun Disord. 1996;29:51-77.

19. Howell P. Signs of developmental stuttering up to eight and at 12 plus. Clin Psychol Rev. 2007;27:287-306.

20. Einarsdottir J, Ingham R. Accuracy of parent identification of stuttering occurrence. Int $\mathrm{J}$ Lang Commun Disord. 2009;12:1-18. 
21. Sawyer J, Yairi E. The effect of sample size on the assessment of stuttering severity. American. J Speech Lang Pathol. 2006;15(1):36-44.

22. Oliveira CMC, Araujo FG, Vocurca MC. Atitudes verbais e não verbais de pais de crianças com queixa de gagueira. Rev Soc Bras Fonoaudiol. 2000;5:3-10.

23. Dehqan A, Bakhtiar M, Panahi SS, Ashayeri $\mathrm{H}$. Relationship between stuttering severity in children and their mother's speaking rate. São Paulo Med J. 2008;126:29-33.

24. Savelkoul EM, Zebrowski PM, Feldstein S, Cole-Harding S. Coordinated interpersonal timing in the conversations of children who stutter and their mothers and fathers. J Fluency Disord. 2007;32:1-32.

25. Millard SK, Nicholas A, Cook FM. Is parent-child interaction therapy effective in reducing stuttering? J Speech Lang Hear Res. 2008;51:636-50.

26. Murphy WP, Yaruss JS, Quesal RW. Enhancing treatment for school-age children who stutter I. Reducing negative reactions through desensitization and cognitive restructuring. J Fluency Disord. 2007;32:121-38.

27. Langevin M, Packman A, Onslow M. Peer responses to stuttering in the preschool setting. Am J Speech Lang Pathol. 2009;18:264-76.

28. Riley GD. Stuttering Severity Instrument for Children and Adults. Austin: Pro Ed; 1994.

29. Ambrose NG, Cox NJ, Yairi, E. The genetic basis of persistence and recovery in stuttering. $\mathrm{J}$ Speech Hear Res. 1997;40:567-80.
30. Andrade CRF. Fluência. In: Andrade CRF, BéfiLopes DM, Fernandes FDM, Wertzner HF. (Editors), ABFW - Teste de linguagem infantil nas áreas de fonologia, vocabulário, fluência e pragmática. Carapicuíba: Pró-Fono; 2004.

31. Dworzynski K, Relington A, Rijsdijk F, Howell P, Plomin R. Genetic etiology in cases of recovered and persistent stuttering in an unselected, longitudinal sample of young twins. Am J Speech Lang Pathol. 2007;16:169-78.

32. Boey RA, Van De Heyning PH, Wuyts FL, Heylen L, Stoop R, De Bodt MS. Awareness and reactions of young stuttering children age 2-7 years old towards their speech disfluency. J Commun Disord. 2009;42(5):334-46.

33. Seery $\mathrm{CH}$, Watkins RV, Mangelsdorf SC, Shigeto A. Subtyping stuttering II: Contributions from language and temperament. J Fluency Disord. 2007;32:197-217.

34. Davis S, Shisca D, Howell, P. Anxiety in speakers who persist and recover from stuttering. J Commun Disord. 2007;40:398-417.

35. Iverach L, O'Brian S, Jones M, Block S, Lincoln M, Harrison E, Hewat S, Menzies RG, Packman A, Onslow M. Prevalence of anxiety disorders among adults seeking speech therapy for stuttering. J Anxiety Disord. 2009;23:928-34.

36. Yairi E. Subtyping stuttering I: a review. J Fluency Disord. 2007;32:165-96.

DOI: 10.1590/S1516-18462010005000116

RECEBIDO EM: 03/03/2010

ACEITO EM: 15/05/2010

Endereço para correspondência:

Cristiane Moço Canhetti de Oliveira

Avenida Hygino Muzzi Filho, 737, Vila Universitária

Marília - SP

CEP:17525-000

E-mail: cmcoliveira@ terra.com.br 